\section{La pathologie au Canada}

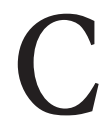

ertaines situations pour le moins alarmantes ont récemment ébranlé le monde de l'anatomopathologie au Canada. Les mauvaises nouvelles qui se succèdent depuis quelques mois préoccupent grandement les anatomopathologistes et minent la confiance de la population à l'endroit de la profession : rapports d'enquête judiciaire sur de faux résultats aux dosages des récepteurs des œstrogènes et de la progestérone dans le dépistage du cancer du sein ${ }^{1}$, enquête publique sur des rapports d'anatomopathologie diagnostique erronés ${ }^{2}$, enquêtes sur de faux diagnostics en laboratoire de pathologie au Nouveau-Brunswick ${ }^{3}$ et plus récemment, taux d'erreur anormalement élevé chez un pathologiste d'Owen Sound, en Ontario ${ }^{4}$. Il n'est bien sûr pas ici question de mettre en doute l'importance de cette discipline. L'examen des tissus en laboratoire d'anatomopathologie constitue la base de la majeure partie des décisions prises dans le domaine des soins de santé et de plus en plus, dans le domaine des thérapeutiques également. Cette discipline permet en outre de faire des liens qui nous aident à mieux comprendre les processus pathologiques. Pendant que nous attendons les recommandations qui émaneront de ces enquêtes, il serait judicieux de réfléchir plus globalement aux raisons pour lesquelles, alors qu'ils jouent un rôle si crucial dans les soins aux patients, les laboratoires d'anatomopathologie semblent avoir autant de plomb dans l'aile, et plus important encore, de trouver les correctifs qui s'imposent.

Sir William Osler a dit «La médecine sera à l'image de la pathologie». Ces mots n'ont pas pris une ride depuis qu'il les a prononcés. Les spécimens soumis au laboratoire de pathologie sont examinés individuellement par le pathologiste; c'est un travail qui demande du temps, qui ne peut être automatisé, qui requiert un sens aigu de l'observation et la capacité d'interpréter les signes observés. La population du Canada a augmenté, notre compréhension des maladies a évolué et plusieurs nouveaux programmes de dépistage et traitements spécialisés, exigeant la confirmation des cibles tissulaires appropriées, ont été mis au point. Avec toutes ces percées, le volume de travail du pathologiste a augmenté et la complexité de chaque cas s'en trouve accrue ${ }^{5}$. Il est fini le temps où une courte note suffisait pour décrire le type de cancer, son étendue et les rebords de la lésion. De nos jours, on ne peut plus se passer des rapports synoptiques ni des échantillons tissulaires complets, des examens exhaustifs au microscope et autres tests complémentaires, dont plusieurs servent à orienter le traitement et permettent de prédire l'issue de la maladie (comme les tests de dépistage des récepteurs des œstrogènes, de la progestérone et du facteur de croissance épidermique humain (HER-2)/neu). Tous ces facteurs font que les laboratoires de pathologie sont débordés. t Contrairement à une clinique, qui peut limiter le nombre de patients reçus chaque jour, le laboratoire de pathologie ne peut pas limiter le nombre de spécimens qu'il reçoit.

Malgré cet environnement où la tâche s'alourdit et se complexifie sans cesse, les laboratoires canadiens continuent de fournir des rapports de pathologie de grande qualité et il serait faux d'affirmer que ces incidents déplorables sont révélateurs d'une situation généralisée au pays. La plupart des pathologistes et technologues canadiens font un travail prodigieux et ce, en dépit des contraintes de temps et autres nombreuses difficultés qu'ils rencontrent, dont une pénurie chronique d'effectifs. L'Association canadienne des pathologistes estime que nous aurons besoin d'environ 500 spécialistes de ce domaine d'ici les ro prochaines années pour répondre aux demandes actuelles $^{6}$ et la Société canadienne de science de laboratoire médical affirme que plus de $50 \%$ des technologistes médicaux prendront leur retraite d'ici $8 \mathrm{ans}^{7}$.

Les erreurs médicales susceptibles d'avoir un impact négatif sur le pronostic d'un patient peuvent se produire n'importe où, surtout dans un système de santé exsangue. En I999, un rapport de l'Institut de médecine des États-Unis pour la sécurité des patients a servi de point de départ à une recension des erreurs médicales enregistrées dans tous les domaines de la médecine aux États-Unis, y compris en pathologie. À la suite de ce rapport, la Commission mixte d'accréditation des organisations de la santé et le Collège américain des pathologistes ont révisé et resserré les normes d'accréditation en médecine de laboratoire en ce qui a trait à la sécurité des patients ${ }^{8}$. On a reconnu le rôle de certaines variables dans les erreurs en laboratoire de pathologie, notamment au chapitre des techniques préanalytiques (p. ex., la durée de la fixation des tissus). Étonnamment, on commence à peine à saisir toute l'importance de standardiser et d'éviter ce type de variation et ces mesures font désormais partie des procédés?.

Au Canada, il existe quelques programmes provinciaux de vérification externe des compétences et d'accréditation des laboratoires : l'Ontario dispose d'un programme de gestion de la qualité des services de laboratoire et la Colombie-Britannique a son Programme d'accréditation diagnostique. Malheureusement, le contrôle des compétences varie d'une province à l'autre. Mais plus important encore, il n'existe pas au Canada d'organisation officiellement responsable de vérifier les compétences techniques à l'échelle nationale. L'Association canadienne des pathologistes a récemment mis sur pied un comité national des normes appliquées à l'immunohistochimie; or, ces initiatives menées sur le terrain font face à une absence de volonté politique et à un manque de fonds flagrant de la part des gouvernements; de plus, ses recommandations ne sont pas exécutoires.

Même si le moment paraît propice à la mise en œuvre d'un programme d'assurance de la qualité pour le volet technique, le système de santé canadien ne s'est pas encore doté d'une approche systématique adéquate en matière d'assurance de la qualité appliquée au volet analytique ou professionnel de l'anatomopathologie. On considère généralement la révision par les pairs comme une importante méthode de prévention des erreurs $^{10}$. Cette mesure peut prendre diverses formes (vérification rétrospective aléatoire, analyse prospective ciblée ou conférences interdépartementales) et s'applique à divers échelons, pour ainsi dire dans tous les départements de pathologie, mais surtout dans les grands centres régionaux, les hôpitaux universitaires et les centres d'oncologie. L'application et le suivi de telles mesures requièrent toutefois des effectifs dont ne disposent pas la plupart 
des laboratoires, particulièrement ceux qui souffrent déjà de graves pénuries de personnel professionnel et technique. Et pourtant, leurs avantages se feraient sentir à plus d'un niveau et l'analyse des erreurs et des discordances permettrait de découvrir que leur origine a moins à voir avec le rendement individuel qu'avec des problèmes d'ordre systémique, c'est-à-dire, ressources humaines, volume de travail, fatigue et surmenage des pathologistes, facteurs liés à une pratique isolée ou restreinte ou absence de ressources de perfectionnement professionnel.

Les laboratoires canadiens ne sont pas les seuls à se plaindre d'un surcroît de travail et d'une pénurie d'effectifs ou à déplorer des erreurs médicales ou une menace à la sécurité des patients ${ }^{11}$. Ils se démarquent toutefois par le fait qu'ils ne disposent pas d'un programme national d'assurance de la qualité comme il en existe au sein du Collège américain des pathologistes, du Collège royal britannique des pathologistes et du Collège royal des pathologistes d'Australasie. Ces organisations supervisent et gèrent un grand nombre d'initiatives en matière d'assurance de la qualité et tout indique que la validité de ces stratégies contribue à faire diminuer le taux d'erreurs ${ }^{12}$. Tous ces programmes prévoient soit des évaluations de la qualité par des organismes indépendants, soit des tests de contrôle des compétences effectués sur une base volontaire, et s'ils ne font pas l'unanimité, les preuves actuelles tendent à confirmer qu'ils contribuent à une meilleure constance dans la préparation des rapports ${ }^{13}$.

Nous partageons tous le même objectif : offrir des services de laboratoire de la plus haute qualité au Canada. Les enquêtes judiciaires et publiques en cours dans différentes régions du pays aboutiront à des recommandations spécifiques; nous pouvons toutefois d'ores et déjà améliorer le système en appliquant 3 mesures d'ordre général.

Premièrement, porter de toute urgence attention aux graves pénuries de personnel pourrait soulager quelque peu les problèmes de manque d'effectifs chroniques et améliorerait le rendement des laboratoires.

Deuxièmement, les administrations hospitalières locales et les ministères provinciaux de la santé devraient sans tarder investir dans des mesures d'assurance de la qualité spécifiques au réseau des laboratoires. On demande beaucoup trop souvent aux laboratoires de mettre eux-mêmes sur pied des initiatives en ce sens, sans tenir compte du fait qu'ils doivent ensuite absorber l'impact des nouveaux programmes cliniques et des nouvelles thérapeutiques ou techniques et autres transformations qui en découlent.

Et en dernier lieu, nous devons créer un organisme national suffisamment pourvu pour promouvoir l'excellence en médecine de laboratoire au Canada. Un tel organisme, comme d'autres, ailleurs dans le monde, ferait le pont entre les programmes d'accréditation provinciaux existants et fournirait des services d'assurance de la qualité dans d'autres régions. Il pourrait également fixer les normes et lignes directrices nationales, établir un système d'assurance de la qualité lié à un programme volontaire de maintien des compétences, coordonner les activités de formation et servir d'organisme conseil pour orienter la planification des effectifs.

Ces trois mesures sont absolument essentielles à l'amélioration et au maintien constants d'un degré élevé de qualité au sein des laboratoires canadiens. Les laboratoires fournissent des renseignements cruciaux pour les soins aux patients et ne serait-ce qu'une seule erreur peut avoir des effets dévastateurs. En accordant la priorité à l'assurance de la qualité et à la sécurité des patients à tous les niveaux, nous arriverons à regagner la confiance des patients, des médecins et de nos pairs à l'endroit de la pathologie.

\section{Kathy Chorneyko MD}

Pathologiste

Directrice médicale adjointe des services de laboratoire

Brant Community Healthcare System

Brantford (Ont.)

\section{Jagdish Butany MBBS MS}

Président, Association canadienne des pathologistes

Professeur, Université de Toronto, Réseau universitaire de la santé Toronto (Ont.)

Avec l'équipe de rédaction de l'éditorial (Paul C. Hébert MD MHSc, Rajendra Kale MD, Matthew B. Stanbrook MD PhD, Barbara Sibbald BJ, Ken Flegel MDCM MSc et Noni MacDonald MD MSc).

Intérêts concurrents : aucuns déclarés pour Kathy Chorneyko et Jagdish $\mathrm{Bu}$ tany. Voir www.cmaj.ca/misc/edboard.shtml pour les déclarations de l'équipe de rédaction de l'éditorial.

\section{RÉFÉRENCES}

I. Weeks C. Io8 women died after botched cancer tests, Newfoundland says. The province prepares to open an inquiry into a medical scandal that has raised doubts about quality control in laboratories across Canada. Globe and Mail. Le ig mars 2008. Accessible à l'adresse : www.theglobeandmail.com/servlet/story/RTGAM.200803I9 .wcancerig/BNStory/specialScienceandHealth/home (consulté le 5 mai 2008).

2. Goudge, the Honourable Justice S.T. Inquiry into Pediatric Forensic Pathology in Ontario. Toronto (Ont.) : The Commission; 2008. Accessible à l'adresse : www .goudgeinquiry.ca (consulté le 5 mai 2008)

3. Police to investigate work of former NB pathologist: thousands of tests under review. Bulletin de nouvelles de la CBC du ir février 2008. Accessible à l'adresse www.cbc.ca/canada/new-brunswick/story/2008/02/II/nb-pathologist.html (consulté le 2 mai 2008).

4. Weeks C. Ontario hospital investigates pathologist. Globe and Mail. Le 30 avril 2008. Accessible à l'adresse : www.theglobeandmail.com/servlet/story/RTGAM .20080430.whospitalo430/BNStory/National/home?cid=al_gam_mostview (consulté le 5 mai 2008).

5. Canadian Association of Pathologists Newsletter. Special Workload Edition 2007 50:8-25.

6. Canadian Association of Pathologists Newsletter, printemps 2007; 50:I8-I9.

7. Canadian Society for Medical Laboratory Science (CSMLS). CMLS reiterates urgent need for long-term human resource strategy for medical laboratory profession. [press release]. Ottawa: CSMLS; le 17 avril 2008. Accessible à l'adresse : www.csmls.org/english/english.htm (consulté le 5 mai 2008).

8. Nakhleh RE. Patient safety and error reduction in surgical pathology. Arch Patho Lab Med 2008; I32:18I-5.

9. Wolff AC, Hammond EH, Schwartz JN, et al. American Society of Clinical Oncology/College of American Pathologists guideline recommendations for human epidermal growth factor receptor 2 testing in breast cancer. Arch Pathol Lab Med 2007;131:18-43.

Io. Association of Directors of Anatomic and Surgical Pathology. Recommendations for quality assurance and improvement in surgical and autopsy pathology. Am Clin Pathol 2006; I2 6:337-40.

II. Misreporting of diagnostic tests in histopathology department at Wrexham Maelor Hospital [press statement]. London (Royaume-Uni) : Royal College of Pathologists; le i6 janvier 2008. Accessible à l'adresse : www.rcpath.org/resources /pdf/pressreleasejano8newales.pdf (consulté le 2 mai 2008).

I2. Raab SS. Improving patient safety through quality assurance. Arch Pathol Lab Med 2006;130:633-7.

I3. Parham DM. Are external quality assessment (EQA) slides schemes a valid tool for the performance assessment of histopathologists? Pathol Res Pract 2005;201:117-2I. 